\title{
Modified FOLFIRINOX for unresectable locally advanced or metastatic gallbladder cancer, a comparison with GEMOX regimen
}

\author{
Xu-Ya Cui ${ }^{1,2,3,4,5,6 \#}$, Xue-Chuan $\mathrm{Li}^{2,3,4,5,6 \#}$, Jiu-Jie Cui ${ }^{4,6,7 \#}$, Xiang-Song Wu ${ }^{1,3,5}$, Lu Zou ${ }^{2,3,4,5,6}$, Xiao-Ling Song ${ }^{1,3,5}$, \\ Tai Ren ${ }^{1,3,5}$, Yi-Di Zhu ${ }^{1,3,5}$, Huai-Feng Li ${ }^{1,3,5}$, Yang Yang ${ }^{2,3,4,5}$, Ke Liu ${ }^{2,3,4,5,6}$, Xu-Sheng Han ${ }^{1,3,5}$, Zi-Yao Jia ${ }^{1,3,5}$, \\ Wen-Guang Wu ${ }^{2,3,4,5,6}$, Xu-An Wang ${ }^{2,3,4,5,6}$, Wei Gong ${ }^{1,3,5}$, Li-Wei Wang ${ }^{4,6,7}$, Mao-Lan Li ${ }^{1,3,5}$, Ying-Bin Liu ${ }^{2,3,4,5,6}$ \\ ${ }^{1}$ Department of General Surgery, Xinhua Hospital Affiliated to Shanghai Jiao Tong University School of Medicine, Shanghai, China; ${ }^{2}$ Department \\ of Biliary-Pancreatic Surgery, Renji Hospital, School of Medicine, Shanghai Jiao Tong University, Shanghai, China; ${ }^{3}$ Shanghai Key Laboratory of \\ Biliary Tract Disease Research, Shanghai, China; ${ }^{4}$ State Key Laboratory of Oncogenes and Related Genes, Shanghai, China; ${ }^{5}$ Shanghai Research \\ Center of Biliary Tract Disease, Shanghai, China; ${ }^{6}$ Shanghai Cancer Institute, Shanghai, China; ${ }^{7}$ Department of Medical Oncology, Renji Hospital, \\ School of Medicine, Shanghai Jiao Tong University, Shanghai, China \\ Contributions: (I) Conception and design: YB Liu, ML Li, LW Wang, XY Cui, XC Li; (II) Administrative support: LW Wang, ML Li, YB Liu; (III) \\ Provision of study materials or patients: W Gong, XS Wu, XA Wang, WG Wu, YD Zhu, HF Li, XL Song; (IV) Collection and assembly of data: XY \\ Cui, JJ Cui, L Zou, Y Yang, K Liu, XS Han, ZY Jia; (V) Data analysis and interpretation: XY Cui, XC Li, L Zou, T Ren; (VI) Manuscript writing: All \\ authors; (VII) Final approval of manuscript: All authors. \\ \#These authors contributed equally to this work. \\ Correspondence to: Ying-Bin Liu, MD, PhD, FACS. Department of Biliary-Pancreatic Surgery, Renji Hospital Affiliated to Shanghai Jiao Tong \\ University School of Medicine, Shanghai 200127, China. Email: laoniulyb@shsmu.edu.cn; Mao-Lan Li, MD, PhD. Department of General Surgery, \\ Xinhua Hospital, Affiliated to Shanghai Jiao Tong University School of Medicine, Shanghai 200092, China. Email: limaolan6@163.com; Li-Wei \\ Wang, MD, PhD. Gastroenterology, Renji Hospital Affiliated to Shanghai Jiao Tong University School of Medicine, Shanghai 200127, China. \\ Email: liweiwang@shsmu.edu.cn.
}

Background: The first-line chemotherapy regimen for advanced gallbladder cancer (GBC) is gemcitabine plus platinum (GP), despite its efficacy is limited. The current investigation is a retrospective study to compare the safety and efficacy between the modified FOLFIRINOX (mFOLFIRINOX) and gemcitabine plus oxaliplatin (GEMOX) as the first-line chemotherapy for unresectable locally advanced or metastatic GBC.

Methods: The data of patients with unresectable locally advanced or metastatic GBC, who were treated with mFOLFIRINOX or GEMOX as the first-line therapy between April 2014 and April 2018 at Xinhua Hospital Affiliated to Shanghai Jiaotong University School of Medicine, were retrieved. This retrospective study evaluated the clinical characteristics, survival outcomes and adverse events.

Results: A total of 44 patients ( $\mathrm{n}=25$ in mFOLFIRINOX, $\mathrm{n}=19$ in GEMOX) were included. There were no significant differences between groups in baseline characteristics. The median progression free survival (mPFS) was 5.0 months in the mFOLFIRINOX group and 2.5 months in the GEMOX group [P=0.021; hazard ratio (HR), 0.499; $95 \% \mathrm{CI}, 0.266$ to 0.937]. The median overall survival (mOS) was 9.5 months in the mFOLFIRINOX group and 7.0 months in the GEMOX group ( $\mathrm{P}=0.019 ; \mathrm{HR}, 0.471 ; 95 \% \mathrm{CI}, 0.239$ to 0.929 ). Disease control rate (DCR) was $76.0 \%$ in the mFOLFIRINOX group and $47.4 \%$ in the GEMOX group $(\mathrm{P}=0.051)$. The rate of grade $3-4$ adverse events was $48 \%$ in the mFOLFIRINOX group and $36.8 \%$ in the GEMOX group $(\mathrm{P}=0.459)$. The incidence of grade 3-4 neutropenia and diarrhea were more common in the mFOLFIRINOX group, while the incidence of grade 3-4 thrombocytopenia and peripheral neuropathy were more common in the GEMOX group.

Conclusions: mFOLFIRINOX might improve the poor prognosis of unresectable locally advanced or metastatic GBC, and the results need to be further verified by prospective clinical studies. 
Keywords: Gallbladder cancer (GBC); modified FOLFIRINOX (mFOLFIRINOX); gemcitabine plus oxaliplatin (GEMOX); chemotherapy

Submitted Dec 16, 2020. Accepted for publication Mar 03, 2021.

doi: $10.21037 / \mathrm{hbsn}-20-846$

View this article at: http://dx.doi.org/10.21037/hbsn-20-846

\section{Introduction}

Gallbladder cancer (GBC) is the most aggressive malignancy of the biliary tract (1). Most patients with GBC are diagnosed in advanced stages and have few chance of surgery (1-3). Chemotherapy is the major palliative treatment for advanced GBC (4). Gemcitabine plus oxaliplatin (GEMOX), a widely accepted first-line chemotherapy regimen for advanced GBC, still resulted in poor overall survival (OS) rates $(5,6)$. Recently, various chemotherapy regimens that try to improve the survival time of advanced GBC have been trialed, but their efficacy still remains unclear $(7,8)$.

The PRODIGE4/ACCORD11 trial has shown that FOLFIRINOX (fluorouracil, leucovorin, irinotecan and oxaliplatin) would significantly improve the OS of metastatic pancreatic cancer (PC), and till now the mOS of the FOLFIRINOX remains the longest in the firstline regimens of PC (9). The chemotherapy regimens for GBC and PC were similar because of the histological, biological and therapeutic (sensitivity to $5-\mathrm{FU}$, platinum, and gemcitabine) similarities $(10,11)$. However, the efficacy and safety of FOLFIRINOX in GBC have not been revealed. This retrospective study was conducted to evaluate the safety and efficacy of modified FOLFIRINOX (mFOLFIRINOX), compared to GEMOX, which is a first-line chemotherapy for unresectable locally advanced or metastatic GBC. We present the following article in accordance with the STROBE reporting checklist (available at https://hbsn.amegroups.com/article/view/10.21037/ hbsn-20-846/rc).

\section{Methods}

The data of patients with unresectable locally advanced or metastatic GBC, treated with mFOLFIRINOX or GEMOX between April 2014 and April 2018 at Xinhua Hospital Affiliated to Shanghai Jiaotong University School of Medicine were retrieved. All diagnoses of gallbladder adenocarcinoma were confirmed via puncture biopsy. The presence of distant metastasis was established using computed tomography (CT) and positron emission tomography-CT (PET-CT). There is currently no definition of unresectable locally advanced GBC. The criteria for unresectability at our institution was defined as those in which surgical resection could not be achieved even by aggressive surgical procedure, including combined vascular resection. In practice, this referred to (I) solid tumor contact with hepatic artery $>180$ degrees; (II) invasion of the tumor to portal vein which is unable to reconstruct; (III) extensive infiltration of the bile duct unable to achieve a curative resection; and (IV) extensive hepatic invasion unable to excise due to insufficient remnant liver volume even after portal vein embolization (12). All individuals were treated with at least one cycle of the MFOLFIRINOX or GEMOX regimen as first-line therapy. The study was conducted in accordance with the Declaration of Helsinki (as revised in 2013). The study was censored on September 29, 2020. Ethical approval was given by the Ethics Committee of Xinhua Hospital Affiliated to Shanghai Jiaotong University School of Medicine (XHEC-D-2020-161). All patients were informed about the purpose and content of the study.

The mFOLFIRINOX regimen was used based on Chines patients' physical condition and on previous regimens used in PC. Patients in the mFOLFIRINOX group treated with 180 to $150 \mathrm{mg} / \mathrm{m}^{2}$ of irinotecan, 85 to $65 \mathrm{mg} / \mathrm{m}^{2}$ of oxaliplatin, $400 \mathrm{mg} / \mathrm{m}^{2}$ of leucovorin and $400 \mathrm{mg} / \mathrm{m}^{2}$ of fluorouracil, proceeded by a continuous fluorouracil infusion of $2,400 \mathrm{mg} / \mathrm{m}^{2}$ spanning $46 \mathrm{~h}$, in a 2 -week schedule. Patients in the GEMOX group treated with $100 \mathrm{mg} / \mathrm{m}^{2}$ of oxaliplatin infused over more than 2 hours on day 1 , and a gemcitabine infusion of $1,000 \mathrm{mg} / \mathrm{m}^{2}$ over $1 \mathrm{~h}$ on days 1 and 8 once every 3 weeks. The same regimen was continued in those who demonstrated a positive response. In case of serious adverse events, treatment was delayed until recovery, but doses were not reduced. Patients discontinued the study in the event of unacceptable toxic effects or evidence of progressive disease, or at their 
request.

\section{Assessments}

Tumors were measured every 4-8 weeks. Tumor response and progression were evaluated according to Response Evaluation Criteria in Solid Tumors (RECIST), version 1.1 (13). All adverse events, monitored from treatment initiation until 28 days after the last treatment, were graded according to the National Cancer Institute Common Terminology Criteria for Adverse Events, version 4.0. The rates of grade 3-4 adverse events between groups were compared. Progression-free survival was calculated from the date of hospitalization until the date of the documentation of disease progression or death due to any cause, which occurred first. OS was calculated from the date of hospitalization until death from any cause.

\section{Statistical analysis}

Qualitative variables were described by frequency and percentage. Quantitative variables were described by the mean plus or minus the standard deviation. Qualitative variables were compared with the use of the chi-square test or Fisher's test. Quantitative variables were compared with the use of a nonparametric (Wilcoxon) test. PFS and OS were estimated with the use of the Kaplan-Meier curve, and were tested by log-rank test. Univariate and multivariate analyses were performed using the Cox proportional hazards regression model to explore prognostic factors for PFS and OS. Regardless of whether statistical differences between groups were observed, the same set of potential confounders was introduced into the Cox regression model for adjustment by the enter method, and data were presented with $95 \%$ confidence intervals. All tests were two-sided, and a $\mathrm{P}$ value of less than 0.05 was considered to indicate statistical significance.

All analyses were performed with intent-to-treat using $\mathrm{SPSS}^{\circledR}$ v 22.0 software (IBM Corp, Armonk, NY, USA).

\section{Results}

\section{Characteristics of the patients}

Between April 2014 and April 2018, a total of 44 patients, received at least one cycle of mFOLFIRINOX or GEMOX for unresectable locally advanced or metastatic GBC at Xinhua Hospital Affiliated to Shanghai Jiaotong University School of Medicine, were included. 25 patients were treated with mFOLFIRINOX (9 males and 16 females, median age 60.8 years), and 19 patients were treated with GEMOX (6 males and 13 females, median age 62.6 years). In the mFOLFIRINOX group, there were 21 patients in clinical T3 stage $(84.0 \%)$ and 4 patients in clinical T4 stage $(16.0 \%)$. In the GEMOX group, there were 17 patients in clinical T3 stage (89.5\%) and 2 patients in clinical T4 stage $(10.5 \%)$ $(\mathrm{P}=0.600)$. Baseline characteristics of patients were similar in the two groups (Table 1).

\section{Efficacy}

The median number of treatment cycle administered was 12 (range, 1 to 21 ) in the mFOLFIRINOX group and 5 (range, 1 to 12 ) in the GEMOX group. The median PFS was 5.0 months (95\% CI, 3.6 to 6.4 months) in the mFOLFIRINOX group and 2.5 months (95\% CI, 1.5 to 3.5 months) in the GEMOX group $(\mathrm{P}=0.021)$ (Figure $1 A)$. The median OS was 9.5 months (95\% CI, 7.4 to 11.6 months) in the mFOLFIRINOX group and 7.0 months (95\% CI, 5.0 to 9.0 months) in the GEMOX group $(\mathrm{P}=0.019)$ (Figure $1 B)$. No patient achieved complete response $(\mathrm{CR})$ in either group. The partial response (PR) was 4 patients $(16.0 \%)$ in the mFOLFIRINOX group and 1 patient $(5.3 \%)$ in the GEMOX group $(\mathrm{P}=0.266)$. The stable disease (SD) was 15 patients $(60.0 \%)$ in the mFOLFIRINOX group and 8 patients $(42.1 \%)$ in the GEMOX group $(\mathrm{P}=0.239)$. The disease control rate (DCR) was $76 \%$ in the mFOLFIRINOX group and $47.4 \%$ in the GEMOX group $(\mathrm{P}=0.051)$ (Table 2).

The univariate and multivariate analyses for PFS and OS were performed. Regardless of whether statistical differences between groups were observed, the same set of potential confounding factors was introduced into the Cox regression model. For PFS, the mFOLFIRINOX regimen compared to GEMOX [hazard ratio (HR), 0.459; 95\% CI, 0.241 to $0.873 ; \mathrm{P}=0.018$, male compared to female (HR, $0.381 ; 95 \%$ CI, 0.172 to $0.843 ; \mathrm{P}=0.017$ ) and metastatic compared to unresectable locally advanced (HR, 2.703; 95\% CI, 1.269 to $5.754 ; \mathrm{P}=0.010$ ) were independent prognostic factors (Table 3). For OS, the mFOLFIRINOX regimen compared to GEMOX (HR, 0.433; 95\% CI, 0.217 to 0.865 ; $\mathrm{P}=0.018$ ), male compared to female (HR, 0.334; 95\% CI, 0.145 to $0.768 ; \mathrm{P}=0.010$ ) and metastatic compared to unresectable locally advanced (HR, 2.393; 95\% CI, 1.102 to 5.194; $\mathrm{P}=0.027$ ) were independent prognostic factor (Table 4).

\section{Safety}


Table 1 Baseline patient characteristics

\begin{tabular}{|c|c|c|c|}
\hline Characteristic & mFOLFIRINOX (n=25) & GEMOX $(n=19)$ & $P$ value \\
\hline \multicolumn{4}{|l|}{ Age (y) } \\
\hline Mean (SD) & $60.8(9.8)$ & $62.6(10.3)$ & \\
\hline$\geq 60$ y, n (\%) & $14(56.0)$ & $10(52.6)$ & 0.824 \\
\hline 0 & $9(36.0)$ & $8(42.1)$ & \\
\hline 1 & $16(64.0)$ & $11(57.9)$ & \\
\hline Clinical T stage, n (\%) & & & 0.600 \\
\hline T3 & $21(84.0)$ & $17(89.5)$ & \\
\hline Unresectable locally advanced & $12(48.0)$ & $6(31.6)$ & \\
\hline Metastatic & $13(52.0)$ & $13(68.4)$ & \\
\hline Liver & $11(44.0)$ & $9(47.4)$ & \\
\hline Pancreas & $1(4.0)$ & $1(5.3)$ & \\
\hline Lung & $1(4.0)$ & $0(0.0)$ & \\
\hline Peritoneum & $2(8.0)$ & $2(10.5)$ & \\
\hline Distant lymph node & $4(16.0)$ & $2(10.5)$ & \\
\hline Median CA19-9 (range, IU/mL) & $69.4(2.7-5,669.0)$ & $76.0(2.9-9,475.0)$ & 0.429 \\
\hline
\end{tabular}

ECOG, Eastern Cooperative Oncology Group.

Table 5 depicts treatment-related grade 3-4 adverse events. The rate of grade 3-4 adverse events was $48 \%$ in the mFOLFIRINOX group and $36.8 \%$ in the GEMOX group $(\mathrm{P}=0.459)$. There was no occurrence of toxic death or emergency admission and febrile neutropenia in either group. Incidences of grade 3-4 neutropenia and diarrhea were higher in the mFOLFIRINOX group, while the incidences of thrombocytopenia and peripheral neuropathy were higher in the GEMOX group.

\section{Discussion}

After a median follow-up approximately 24 months, the median PFS and median OS of the mFOLFIRINOX group was 5.0 and 9.5 months, respectively. These outcomes appear more favorable compared with the GEMOX group (median PFS 2.5 months and median OS 7.0 months). Moreover, GEMOX regimen efficacy noted in our study corresponded well with previous studies on gemcitabine- based therapies for advanced GBC (median PFS of 3.0 to 6.2 months and median OS of 6.2 to 12.1 months) (4,14-16).

Chemotherapy has been widely used in the treatment of GBC for a long time (17). What's more, National Comprehensive Cancer Network has provided options for advanced GBC treatment: GEMOX and other regimens which includes 5-fluorouracil, oxaliplatin, cisplatin, and capecitabine, etc. (7), but till now the OS rates of advanced GBC remained poor. Meanwhile, other regimens such as gemcitabine plus S-1 (GS), gemcitabine, cisplatin, and nab-paclitaxel had achieved satisfactory results in clinical trials $(8,18)$. However, the evidence remains limited to form recommendations for a standard chemotherapy regimen. And most of these reports were limited by tumor heterogeneity or lack of survival comparison with standard chemotherapy.

Previous studies have shown that FOLFIRINOX can prolong the OS of patients with advanced $\mathrm{PC}$ and National Comprehensive Cancer Network recommends 
FOLFIRINOX as the first-line treatment option for PC treatment (9). The PRODIGE4/ACCORD 11 trial has shown that FOLFIRINOX could significantly improve the OS of metastatic PC, and till now the mOS of the FOLFIRINOX remains the longest in the firstline regimens of PC. The treatments for GBC and PC were similar because of the histological, biological and therapeutic (sensitivity to 5-FU, platinum, and gemcitabine) similarities $(10,11)$. This retrospective study was performed to analyze the efficacy and safety of mFOLFIRINOX versus GEMOX which are both first-line chemotherapies

A

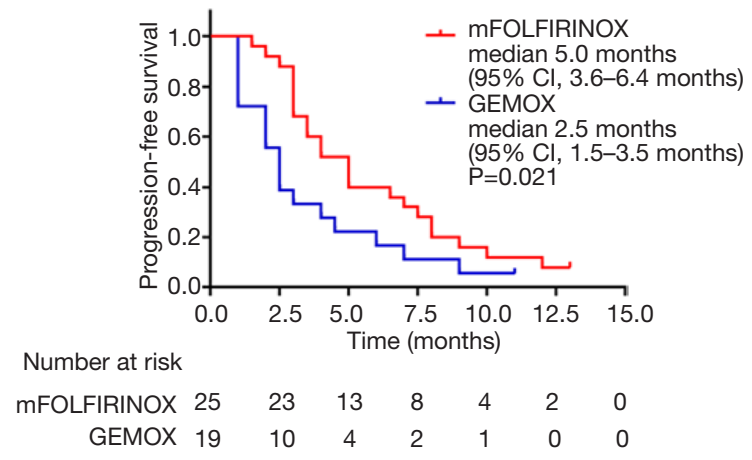

B

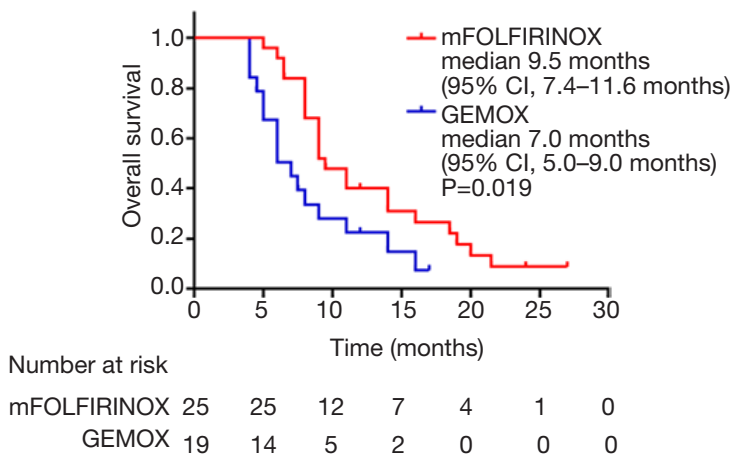

Figure 1 Progression-free survival (A) and Overall survival (B) in patients receiving mFOLFIRINOX $(n=25)$ and GEMOX $(n=19)$. for patients with unresectable locally advanced or metastatic GBC. Patients' regimen selections depended on their personal wishes and the recommendations of physicians. There is no difference between the price of two chemotherapeutic regimens. And the dose of mFOLFIRINOX used in this study was based on the dose of advanced PC and general patient condition to maintain efficacy and avoid the high rate of adverse events $(19,20)$. The results of this study showed that DCR was $76 \%$ in the mFOLFIRINOX group and was $47.4 \%$ in the GEMOX group $(\mathrm{P}=0.051)$. However, better chemotherapeutic effects were achieved in the mFOLFIRINOX group (Figure $2 A$ ). In addition, 2 unresectable locally advanced patients underwent the surgical resection after multidisciplinary treatment (MDT) (Figure 2B), while none of the 19 patients had the opportunity to undergo the surgical resection in the GEMOX group. The results of multivariate analyses showed that treatment regimens, metastasis and sex were poor prognostic factors for PFS and OS, which was similar with previous studies that metastasis and gender were independent poorly prognostic factors for $\operatorname{GBC}(2,3)$.

The rate of grade $3-4$ adverse events was $48 \%$ in the mFOLFIRINOX group and $36.8 \%$ in the GEMOX group $(\mathrm{P}=0.459)$. The safety of mFOLFIRINOX was associated with a higher incidence of grade 3 or 4 neutropenia and diarrhea. Irinotecan and high doses of fluorouracil might be the cause. And the safety of GEMOX was associated with higher incidence of grade 3 or 4 thrombocytopenia and peripheral neuropathy and were consistent with previous studies (5).

However, there are several limitations in this study. First, it is a retrospective study. It was subject to patient's selection bias, the doctor's recommendation, and its conclusions were observational. In addition, during the study period, limited sample size and unrecognized changes in practice might limit the analysis of treatment effects. Moreover, the inclusion of 6 variables in this study might lead to unstable results of the multivariate analysis. As a result of these

Table 2 Best tumor response based on the response evaluation criteria in solid tumors (RECIST) 1.1

\begin{tabular}{lccc}
\hline Response & mFOLFIRINOX $(\mathrm{n}=25)$ & GEMOX $(\mathrm{n}=19)$ & P value \\
\hline Complete response, $\mathrm{n}(\%)$ & $0(0.0)$ & $0(0.0)$ & - \\
Partial response, $\mathrm{n}(\%)$ & $4(16.0)$ & $1(5.3)$ & 0.266 \\
Stable disease, $\mathrm{n}(\%)$ & $15(60.0)$ & $8(42.1)$ & 0.239 \\
Progressive disease, $\mathrm{n}(\%)$ & $6(24.0)$ & $10(52.6)$ & 0.051 \\
\hline
\end{tabular}


Table 3 Univariate and multivariate analysis for PFS

\begin{tabular}{|c|c|c|c|c|}
\hline Variables & \multicolumn{2}{|c|}{ Univariate analysis } & \multicolumn{2}{|c|}{ Multivariate analysis } \\
\hline \multicolumn{5}{|l|}{ Treatment regimen } \\
\hline mFOLFIRINOX vs. GEMOX & $0.499(0.266-0.937)$ & 0.031 & $0.459(0.241-0.873)$ & 0.018 \\
\hline \multicolumn{5}{|l|}{ Age } \\
\hline \multicolumn{5}{|l|}{ Sex } \\
\hline Male vs. female & $0.474(0.231-0.972)$ & 0.042 & $0.381(0.172-0.843)$ & 0.017 \\
\hline \multicolumn{5}{|l|}{ ECOG } \\
\hline 1 vs. 0 & $1.160(0.606-2.221)$ & 0.655 & $1.256(0.592-2.666)$ & 0.553 \\
\hline \multicolumn{5}{|l|}{ CA 19-9 } \\
\hline$\geq 37$ vs. $<37$ & $1.323(0.663-2.642)$ & 0.427 & $0.650(0.280-1.513)$ & 0.318 \\
\hline
\end{tabular}

Table 4 Univariate and multivariate analysis for OS

\begin{tabular}{|c|c|c|c|c|}
\hline \multirow{2}{*}{ Variables } & \multicolumn{2}{|c|}{ Univariate analysis } & \multicolumn{2}{|c|}{ Multivariate analysis } \\
\hline & $\mathrm{HR}(95 \% \mathrm{Cl})$ & $P$ value & $\mathrm{HR}(95 \% \mathrm{Cl})$ & $P$ value \\
\hline \multicolumn{5}{|l|}{ Treatment regimen } \\
\hline mFOLFIRINOX vs. GEMOX & $0.471(0.239-0.929)$ & 0.030 & $0.433(0.217-0.865)$ & 0.018 \\
\hline \multicolumn{5}{|l|}{ Age } \\
\hline \multicolumn{5}{|l|}{ Sex } \\
\hline Male vs. female & $0.434(0.206-0.914)$ & 0.028 & $0.334(0.145-0.768)$ & 0.010 \\
\hline \multicolumn{5}{|l|}{ ECOG } \\
\hline 1 vs. 0 & $1.314(0.661-2.615)$ & 0.436 & $1.632(0.720-3.701)$ & 0.241 \\
\hline \multicolumn{5}{|l|}{ CA 19-9 } \\
\hline$\geq 37$ vs. $<37$ & $0.641(0.586-2.386)$ & 0.641 & $0.521(0.222-1.221)$ & 0.134 \\
\hline
\end{tabular}

$\mathrm{P}<0.05$ was considered statistically significant. OS, overall survival; HR hazard ratio; ECOG, Eastern Cooperative Oncology Group. 
Table 5 Most common grade 3-4 adverse events occurring in more than $5 \%$ of patients in the safety population

\begin{tabular}{|c|c|c|c|}
\hline & mFOLFIRINOX grade 3-4, n (\%) & GEMOX grade 3-4, n (\%) & $P$ value \\
\hline \multicolumn{4}{|l|}{ Hematological } \\
\hline Neutropenia & $7(28.0)$ & $3(15.8)$ & \\
\hline Thrombocytopenia & $2(8.0)$ & $4(21.1)$ & \\
\hline \multicolumn{4}{|l|}{ Non-hematological } \\
\hline Nausea & $2(8.0)$ & $1(5.3)$ & \\
\hline Vomiting & $2(8.0)$ & $2(10.5)$ & \\
\hline Anorexia & $1(4.0)$ & $1(5.3)$ & \\
\hline
\end{tabular}

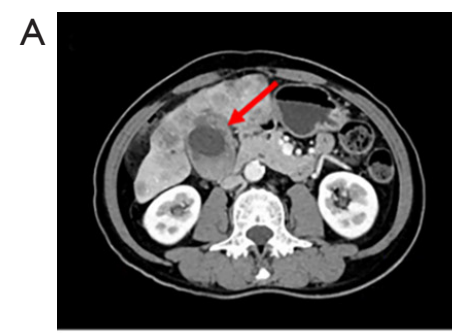

Pre mFOLFIRINOX

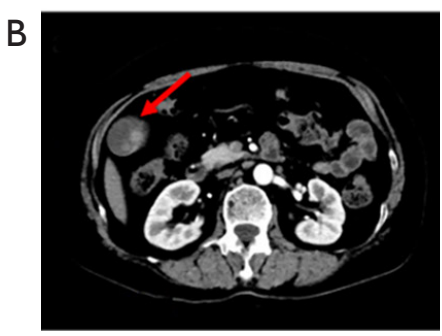

Pre mFOLFIRINOX

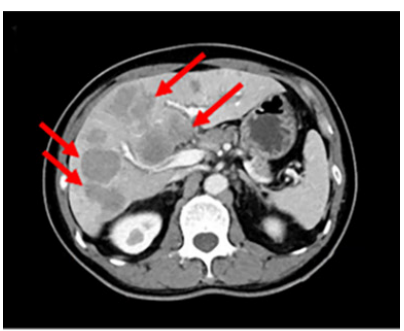

Pre mFOLFIRINOX

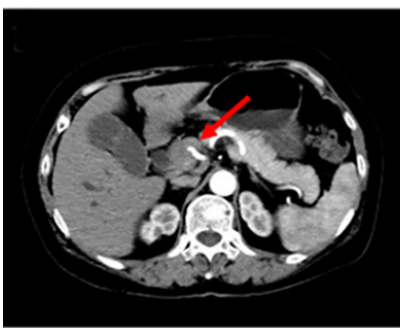

Pre mFOLFIRINOX

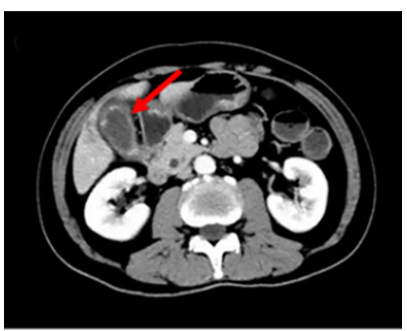

Post mFOLFIRINOX

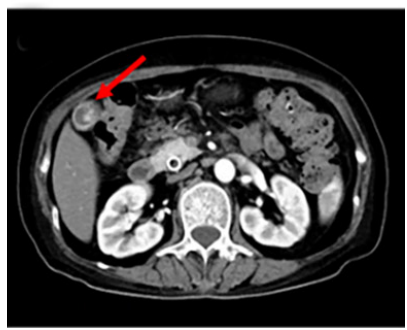

Post mFOLFIRINOX

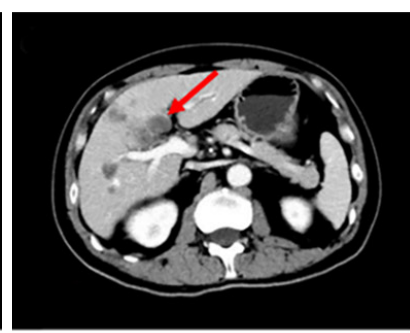

Post mFOLFIRINOX

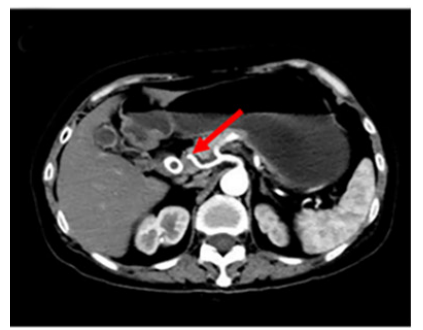

Post mFOLFIRINOX

Figure 2 Effect of modified FOLFIRINOX (mFOLFIRINOX) treatment. (A) Figures are representative pre- and post-mFOLFIRINOX treatment in a 64-year-old male with metastatic gallbladder cancer. After 4 cycles of mFOLFIRINOX treatment, primary tumor (red arrows) and extensive metastatic lesions involving liver (red arrowheads) became markedly reduced compared with those in pre-mFOLFIRINOX treatment CT images. (B) Figures are representative pre- and post-mFOLFIRINOX treatment in a 61-year-old female with unresectable locally advanced gallbladder cancer. After 4 cycles of mFOLFIRINOX treatment, primary tumor and para-hepatic artery lymph node markedly reduced and gaps around the hepatic artery were observed. R0 resection was successfully achieved after MDT consultation. 
limitations, our findings should be carried out in a very discreet manner. Further, randomized prospective trials are necessary to be carried out to determine the efficacy of mFOLFIRINOX regimen to further evaluate the impact on the OS of GBC patients.

\section{Conclusions}

mFOLFIRINOX might improve the poor prognosis of unresectable locally advanced or metastatic GBC, and the results need to be further verified by prospective clinical studies.

\section{Acknowledgments}

The author thanks the members of the Shanghai Key Laboratory of Biliary Tract Diseases for their assistance in data entry. Secondly, thanks to Dr. Xi Zhang at Xinhua Hospital Affiliated to Shanghai Jiao Tong University School of Medicine for revising this manuscript.

Funding: This work was supported by the National Natural Science Foundation of China (grant numbers 31620103910, 81874181, 82073206, 81773043); the National Science and Technology Major Projects for "Major New Drugs Innovation and Development" (grant number 2019ZX09301158); Emerging Frontier Projects of Shanghai-Level Hospitals (grant number SHDC12018107); "Shuguang Program" supported by Shanghai Education Development Foundation and Shanghai Municipal Education Commission (grant number 20SG14); the Program of Shanghai Academic Research Leader (grant number 19XD1422700); the Peak Plateau Discipline Construction Project of Shanghai Jiaotong University School of Medicine (grant number 20181808); Clinical Research Program of Xinhua Hospital (19XHCR3D).

\section{Footnote}

Reporting Checklist: The authors have completed the STROBE reporting checklist. Available at https://hbsn. amegroups.com/article/view/10.21037/hbsn-20-846/rc

Data Sharing Statement: Available at https://hbsn. amegroups.com/article/view/10.21037/hbsn-20-846/dss

Conflicts of Interest: All authors have completed the ICMJE uniform disclosure form (available at https://hbsn. amegroups.com/article/view/10.21037/hbsn-20-846/coif).
The authors have no conflicts of interest to declare.

Ethical Statement: The authors are accountable for all aspects of the work in ensuring that questions related to the accuracy or integrity of any part of the work are appropriately investigated and resolved. The study was conducted in accordance with the Declaration of Helsinki (as revised in 2013). Ethical approval was given by the Ethics Committee of Xinhua Hospital Affiliated to Shanghai Jiaotong University School of Medicine (XHEC-D-2020-161). All patients were informed about the purpose and content of the study.

Open Access Statement: This is an Open Access article distributed in accordance with the Creative Commons Attribution-NonCommercial-NoDerivs 4.0 International License (CC BY-NC-ND 4.0), which permits the noncommercial replication and distribution of the article with the strict proviso that no changes or edits are made and the original work is properly cited (including links to both the formal publication through the relevant DOI and the license). See: https://creativecommons.org/licenses/by-nc-nd/4.0/.

\section{References}

1. Li M, Zhang Z, Li X, et al. Whole-exome and targeted gene sequencing of gallbladder carcinoma identifies recurrent mutations in the ErbB pathway. Nat Genet 2014;46:872-6.

2. Baiu I, Visser B. Gallbladder Cancer. JAMA 2018;320:1294.

3. Li M, Liu F, Zhang F, et al. Genomic / mutations promote PD-L1-mediated immune escape in gallbladder cancer: a whole-exome sequencing analysis. Gut 2019;68:1024-33.

4. Lamarca A, Barriuso J, McNamara MG, et al. Molecular targeted therapies: Ready for "prime time" in biliary tract cancer. J Hepatol 2020;73:170-85.

5. Jang JS, Lim HY, Hwang IG, et al. Gemcitabine and oxaliplatin in patients with unresectable biliary cancer including gall bladder cancer: a Korean Cancer Study Group phase II trial. Cancer Chemother Pharmacol 2010;65:641-7.

6. Valle J, Wasan H, Palmer DH, et al. Cisplatin plus gemcitabine versus gemcitabine for biliary tract cancer. $\mathrm{N}$ Engl J Med 2010;362:1273-81.

7. Primrose JN, Fox RP, Palmer DH, et al. Capecitabine compared with observation in resected biliary tract cancer (BILCAP): a randomised, controlled, multicentre, phase 3 
study. Lancet Oncol 2019;20:663-73.

8. Morizane C, Okusaka T, Mizusawa J, et al. Combination gemcitabine plus $\mathrm{S}-1$ versus gemcitabine plus cisplatin for advanced/recurrent biliary tract cancer: the FUGABT (JCOG1113) randomized phase III clinical trial. Ann Oncol 2019;30:1950-8.

9. Conroy T, Desseigne F, Ychou M, et al. FOLFIRINOX versus gemcitabine for metastatic pancreatic cancer. $\mathrm{N}$ Engl J Med 2011;364:1817-25.

10. Phelip JM, Edeline J, Blanc JF, et al. Modified FOLFIRINOX versus CisGem first-line chemotherapy for locally advanced non resectable or metastatic biliary tract cancer (AMEBICA)-PRODIGE 38: Study protocol for a randomized controlled multicenter phase II/III study. Dig Liver Dis 2019;51:318-20.

11. Glimelius B, Hoffman K, Sjödén PO, et al. Chemotherapy improves survival and quality of life in advanced pancreatic and biliary cancer. Ann Oncol 1996;7:593-600.

12. Kato A, Shimizu H, Ohtsuka M, et al. Surgical resection after downsizing chemotherapy for initially unresectable locally advanced biliary tract cancer: a retrospective singlecenter study. Ann Surg Oncol 2013;20:318-24.

13. Eisenhauer EA, Therasse P, Bogaerts J, et al. New response evaluation criteria in solid tumours: revised RECIST guideline (version 1.1). Eur J Cancer 2009;45:228-47.

14. Edeline J, Benabdelghani M, Bertaut A, et al. Gemcitabine and Oxaliplatin Chemotherapy or Surveillance in Resected Biliary Tract Cancer (PRODIGE 12-ACCORD 18-UNICANCER GI): A Randomized Phase III Study. J Clin Oncol 2019;37:658-67.

15. André T, Tournigand C, Rosmorduc O, et al. Gemcitabine combined with oxaliplatin (GEMOX) in advanced biliary tract adenocarcinoma: a GERCOR study. Ann Oncol 2004;15:1339-43.

16. Woo SM, Lee SH, Yoo JW, et al. A Multicenter Phase II Trial of Gemcitabine Plus Oxaliplatin in Unresectable Gallbladder Cancer. Gut Liver 2013;7:594-8.

17. Song X, Hu Y, Li Y, et al. Overview of current targeted therapy in gallbladder cancer. Signal Transduct Target Ther 2020;5:230.

18. Shroff RT, Javle MM, Xiao L, et al. Gemcitabine, Cisplatin, and nab-Paclitaxel for the Treatment of Advanced Biliary Tract Cancers: A Phase 2 Clinical Trial. JAMA Oncol 2019;5:824-30.

19. Li X, Ma T, Zhang Q, et al. Modified-FOLFIRINOX in metastatic pancreatic cancer: A prospective study in Chinese population. Cancer Lett 2017;406:22-6.

20. Stein SM, James ES, Deng Y, et al. Final analysis of a phase II study of modified FOLFIRINOX in locally advanced and metastatic pancreatic cancer. Br J Cancer 2016;114:737-43.
Cite this article as: Cui XY, Li XC, Cui JJ, Wu XS, Zou L, Song XL, Ren T, Zhu YD, Li HF, Yang Y, Liu K, Han XS, Jia ZY, Wu WG, Wang XA, Gong W, Wang LW, Li ML, Liu YB. Modified FOLFIRINOX for unresectable locally advanced or metastatic gallbladder cancer, a comparison with GEMOX regimen. HepatoBiliary Surg Nutr 2021;10(4):498-506. doi: 10.21037/hbsn-20-846 